\title{
ChemComm
}

\section{Height dependent molecular trapping in stacked cyclic porphyrin nanorings $\dagger$}

Cite this: Chem. Commun., 2014, 50, 7332

Received 9th April 2014,

Accepted 18th May 2014

DOI: $10.1039 / \mathrm{c} 4 \mathrm{cc0} 02629 \mathrm{~b}$

www.rsc.org/chemcomm

\author{
Maria B. Wieland, ${ }^{a}$ Luis M. A. Perdigão, ${ }^{a}$ Dmitry V. Kondratuk, ${ }^{b}$ James N. O'Shea, ${ }^{a}$ \\ Harry L. Anderson ${ }^{b}$ and Peter H. Beton ${ }^{\star a}$
}

\begin{abstract}
Stacked layers of cyclic porphyrin nanorings constitute nanoscale receptacles with variable height and diameter which preferentially adsorb sublimed molecules. Using scanning tunnelling microscopy we determine the filling capacity of these nanoring traps, and the dependence of adsorbate capture on stack height and diameter.
\end{abstract}

The formation of arrays of nanopores which have the potential to trap and organise adsorbed guest molecules has been widely studied as a route to combine spatial organisation and chemical functionalization on a surface. ${ }^{1-5}$ This has been successfully achieved using nanoporous templates stabilised by hydrogen bonding, metal co-ordination and covalent coupling, both in solution $^{6-11}$ and in vacuum. ${ }^{1,2,12}$ In most cases the nanoporous networks have a height corresponding to a single monolayer but here we explore the capture of molecules within a trap formed by stacked layers of porphyrin nanorings ${ }^{13-15}$ with diameters up to $10 \mathrm{~nm}$ and heights of $\sim 1 \mathrm{~nm}$. We find significant differences between the statistics of the capture of sublimed $\mathrm{C}_{60}$ molecules depending on both height and diameter of the traps.

A schematic diagram of the cyclic porphyrin nanoring is shown in Fig. 1a. Each porphyrin macrocycle is linked to two neighbouring porphyrins by butadiyne linker groups and the nanorings are synthesised using a templating technique. ${ }^{13,14}$ In this study we have investigated $\boldsymbol{c}$-P12 and $\boldsymbol{c}$-P24 which have, respectively, 12 and 24 porphyrin groups, and diameters, assuming a circular conformation, of approximately $5 \mathrm{~nm}$ and $10 \mathrm{~nm}$. In previous work we have shown that the nanorings may be deposited on a surface held under vacuum conditions using electrospray deposition. ${ }^{16,17}$ The smaller $\boldsymbol{c}$-P12 adopts a nearcircular conformation (diameter $\sim 5 \mathrm{~nm}$ ) with monolayer height in which the constituent porphyrin groups lie parallel to a supporting substrate $(\mathrm{Au}(111))$. The larger $\boldsymbol{c}$-P24 shows a

\footnotetext{
${ }^{a}$ School of Physics and Astronomy, University of Nottingham, Nottingham, NG72RD, UK. E-mail: peter.beton@nottingham.ac.uk

${ }^{b}$ Department of Chemistry, University of Oxford, Chemistry Research Laboratory, Oxford, OX1 3TA, UK

$\dagger$ Electronic supplementary information (ESI) available: Sample preparation. See DOI: $10.1039 / \mathrm{c} 4 \mathrm{cc} 02629 \mathrm{~b}$
}

more complex behaviour with significant deviations from a circular conformation due to the inherent flexibility of the nanorings. In addition we observe columnar stacks of $\boldsymbol{c}$-P24 up to 4 layers high; the presence of the stacked rings is controlled by the solvent chosen for use in the electrospray process, implying that these nanostructures are formed prior to deposition. We have argued that this is due to aggregations stabilised by $\pi-\pi$ interactions ${ }^{15,18}$ The variable height of the columnar stacks facilitates a study of the influence of the vertical dimension (perpendicular to the substrate) on the capture of guest molecules by a supramolecular trap.

In the experiments described below we first prepare a $\mathrm{Au}(111)$ surface under ultra-high vacuum (UHV) conditions. Porphyrin nanorings are then deposited using electrospray and, after characterisation of the surface using a scanning tunnelling microscope (STM) housed within the UHV system, $\mathrm{C}_{60}$ is deposited by sublimation. The experimental procedures are similar to those adopted in our previous work ${ }^{13-15}$ and are described fully in ESI. $\dagger$

Fig. 1b shows an STM image of the surface following the deposition of $\boldsymbol{c}$-P24 followed by 0.3 monolayers (ML) of $\mathrm{C}_{60}$. The nanorings are clearly resolved as cyclic structures with characteristic dimensions of $10 \mathrm{~nm}$. As observed in our previous work the nanorings are not perfectly circular but undergo distortions due to their flexibility; in some cases the distortion is extreme - for example the nanoring near the right edge of Fig. 1b. The nanorings have different heights as revealed by their different apparent brightness. In this particular image there are three bright rings corresponding to a stack of three nanorings and two with lower contrast which correspond to a height of two stacked rings. In addition there is one ring which has the height of a single monolayer in the top right corner which is difficult to resolve in this figure due to the overall height contrast range; under these scanning conditions this monolayer nanoring has a slight negative contrast. The approximate frequency of occurrence of stacks of 1,2 and 3 rings is $1: 1: 2$. In common with our previous observations many nanorings are observed either overlapping or tangential to Au terrace step edges.

Also apparent in Fig. $1 \mathrm{~b}$ are rows of $\mathrm{C}_{60}$ molecules which are predominantly adsorbed either along step edges on the 
a
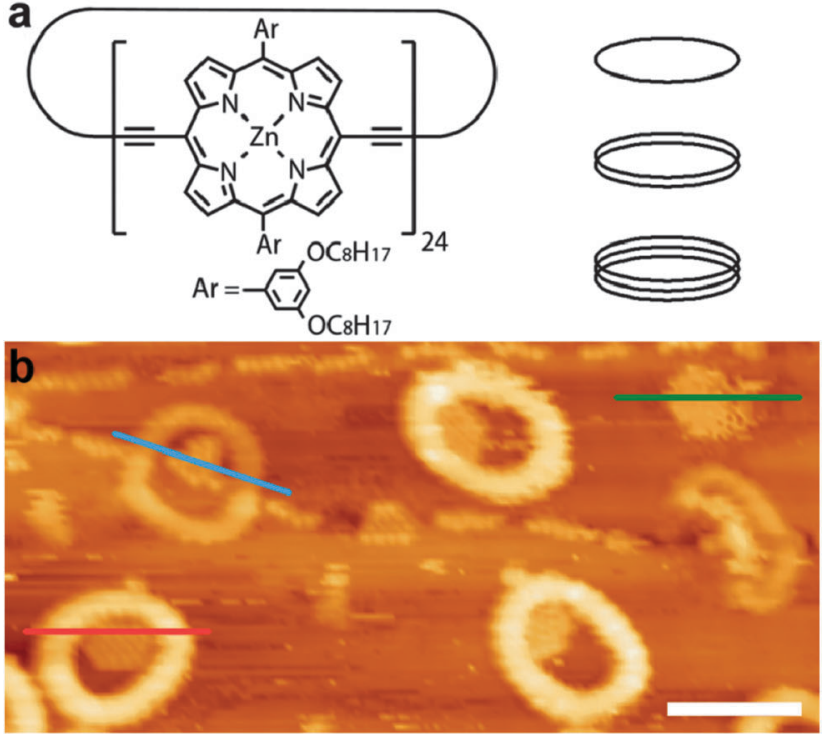

C
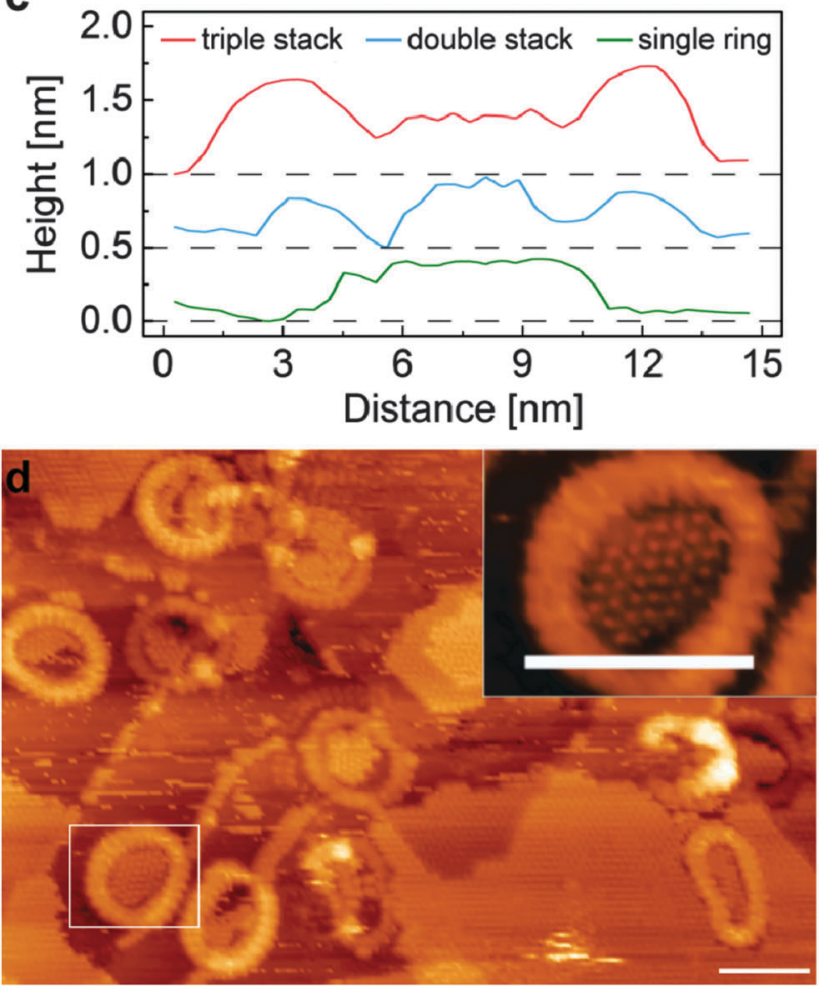

Fig. 1 (a) Chemical structure of $\boldsymbol{c}$-P24 (left); schematic of a single, a double stacked, and a triple stacked nanoring (right); (b) STM image (-1.7V, $0.03 \mathrm{nA})$ of c-P24 porphyrin nanorings deposited via electrospray deposition and subsequently sublimed $0.3 \mathrm{ML} \mathrm{C}_{60}$; profile lines indicated; scale bar $10 \mathrm{~nm}$; (c) profiles through porphyrin nanorings of variable stacking height partially filled with $\mathrm{C}_{60}$; (d) STM image $(-1.7 \mathrm{~V}, 0.03 \mathrm{nA}$ ) of $\boldsymbol{c}$-P24 porphyrin nanorings deposited via electrospray deposition and subsequently sublimed $0.7 \mathrm{ML}$ $\mathrm{C}_{60}$; inset: zoom of marked ring with showing positions of close-packed $\mathrm{C}_{60}$ molecules; scale bar $10 \mathrm{~nm}$.

$\mathrm{Au}(111)$ surface, or as islands nucleated within the nanorings. In the lower left of the image a triple height nanoring is clearly resolved and is identified by the red line along which a height profile is extracted and shown in Fig. 1c. A height profile is also shown for a double height nanoring (upper left; blue line profile); this nanoring straddles a step edge of the $\mathrm{Au}(111)$ surface. The height profile (green line) of the monolayer nanoring is also shown in Fig. 1c. In each of these nanorings there is captured $\mathrm{C}_{60}$ which has an effective height, from the line profiles, of $\sim 0.4 \mathrm{~nm}$ and is approximately the same for each ring. This effective height for the $\mathrm{C}_{60}$ is close to that expected from previous studies. ${ }^{19}$ In contrast the height of the enclosing nanoring differs significantly; for the triple height ring we measure $0.65 \mathrm{~nm}$, for the double height ring, $0.3 \mathrm{~nm}$ and for the monolayer we measure a depression of $\sim 0.1 \mathrm{~nm}$. The difference in height due to the addition of each layer is close to the value of $0.34 \mathrm{~nm}$ measured previously, but the absolute heights of the nanorings are lower by approximately $0.2 \mathrm{~nm}$ as compared with our previous work. We attribute this systematic difference to variations in the termination of the STM tip as compared with our previous work; in particular it is possible that in these experiments $\mathrm{C}_{60}$ is transferred to the tip.

For this $\mathrm{C}_{60}$ coverage, the islands within the three triple height nanoring stacks occupy approximately half the available enclosed area, and share a common boundary with the inner edge of the nanorings. This indicates that the internal nanoring edge is associated with the nucleation of the $\mathrm{C}_{60}$ islands, although, as discussed below, we resolve a small gap between the nanoring and the enclosed $\mathrm{C}_{60}$.

A slightly different behaviour is observed for the double height nanoring identified in Fig. 1c where the nucleation has likely occurred at the Au step edge and the resulting island is wholly enclosed within the nanoring; we also see many double height stacks where the growth is nucleated at the nanoring edge, similar to the triple height nanorings. Interestingly the $\mathrm{C}_{60}$ island in the monolayer height island in Fig. 1b occupies a higher fraction of the area enclosed by the nanoring than is observed for the double and triple layer stacks. We also note that there is evidence of mobile adsorbed material on this surface which gives rise to streaking in the image.

In Fig. 1d we show an image acquired after further deposition of $\mathrm{C}_{60}$. In addition to the $\mathrm{C}_{60}$ trapped within the rings and step edges we also see the growth of large fullerene islands at this coverage $(0.7 \mathrm{ML})$. The typical island size within the nanoring is also larger. The inset to Fig. 1d shows a zoomed image of a nanoring with an enclosed $\mathrm{C}_{60}$ island. In this image it is possible to resolve both individual $\mathrm{C}_{60}$ molecules and also the porphyrin sub-units within the nanoring; the latter are associated with the regular contrast variation around the perimeter of the nanoring and it is possible to confirm that, as expected, there are 24 groups. The images also show that $\mathrm{C}_{60}$ molecules within the nanoring adopt the expected hexagonal close packed structure. In addition, we can clearly resolve in this image a low contrast gap between the $\mathrm{C}_{60}$ molecules and the centre of the porphyrin groups. This separation is typically $1.5 \mathrm{~nm}$ and its presence shows that there is an excluded area for $\mathrm{C}_{60}$ within the ring.

After a series of sequential depositions the variation of the effective coverage of $\mathrm{C}_{60}$ inside and outside the nanorings is determined (124 nanorings analysed over fifteen $100 \mathrm{~nm}^{2}$ images). First we note the linear dependence of $\mathrm{C}_{60}$ coverage outside the 


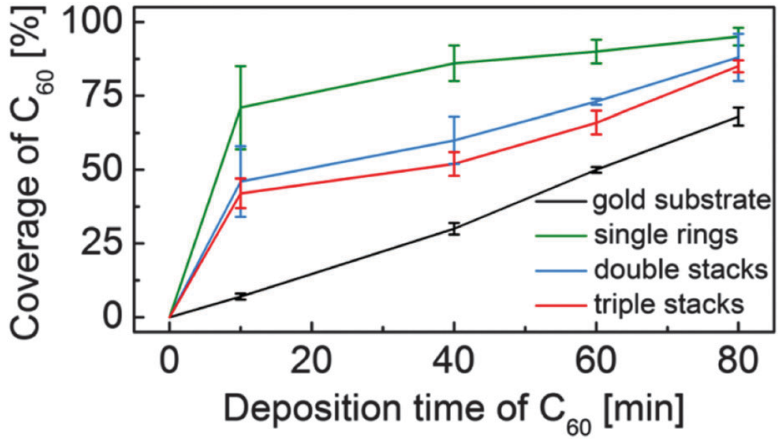

Fig. 2 Coverage of $C_{60}$ inside and outside the porphyrin nanorings with increasing $\mathrm{C}_{60}$ deposition time.

nanorings with cumulative deposition time. In determining this value we first exclude from the overall area within an image those parts of the surface covered, or enclosed by nanorings. The proportion of this fraction of the surface covered by $\mathrm{C}_{60}$ is then plotted in Fig. 2. The coverage of $\mathrm{C}_{60}$ within the nanorings is determined as a fraction of area of the enclosed $\mathrm{C}_{60}$ island divided by the pore area of the individual ring (which varies due to shape distortion). For the ring internal area we take the dark contrast area enclosed by the inner perimeter of the nanoring. The pore size ranges from $34-38 \mathrm{~nm}^{2}$, equivalent to a circle with a radius of $\sim 3.4 \pm 0.2 \mathrm{~nm}$. This is consistent with the maximum number of trapped $\mathrm{C}_{60}$ molecules, which we find to be 37 and represents the capacity of the nanoring.

From Fig. 2 it is clear that there is an enhanced probability of fullerene adsorption within the nanorings. In addition, the capture probability is highest for the monolayer rings, with the higher, stacked rings having a capture probability intermediate between that of the monolayers and the surrounding gold surface. These observations are consistent with an enhanced nucleation rate for $\mathrm{C}_{60}$ island formation within the nanorings which might arise, for example, from an enhanced residence time of a molecule within the nanoring. This might be expected due to the interaction of the $\mathrm{C}_{60}$ with the internal edges of the nanoring and is also consistent with the presence of a barrier for molecules to diffuse over the nanoring and escape from the local trapped region. We further suggest that that the reduction of relative coverage for higher, stacked rings is due to a larger barrier which molecules must overcome to enter a nanopore. Note that the density within nanorings of all heights is significantly greater than the surrounding region, particularly at low coverage, implying that at least some of the captured molecules must be incident elsewhere on the surface and diffuse before becoming trapped in the nanoring.

The capacity of $\boldsymbol{c}$-P24 is significantly less than that expected from the geometric radius of $\boldsymbol{c}-\mathbf{P 2 4}, 5 \mathrm{~nm}$. The effective radius is $\sim 3.4 \mathrm{~nm}$, and the difference, $1.6 \mathrm{~nm}$, is very close to the length of the solubilising groups attached to the porphyrin macrocycles. We therefore suggest that these sidegroups are adsorbed in a quasi-linear conformation which spreads radially across the surface giving rise to a region where it is not energetically favourable for $\mathrm{C}_{60}$ islands to grow. This in-plane conformation of the sidegroups is consistent with our previous observations on the packing of linear analogue polymers. ${ }^{17}$

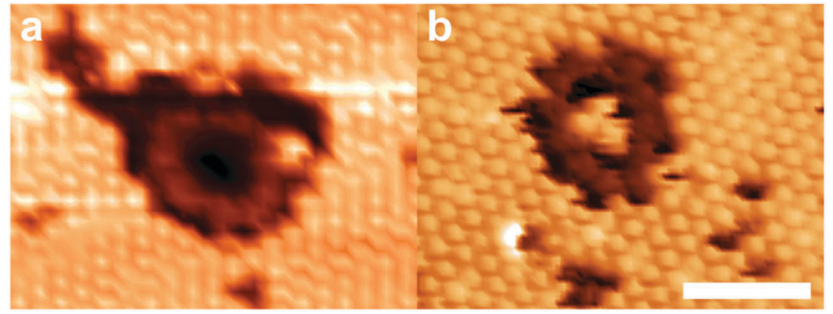

Fig. 3 STM images ( $-1.7 \mathrm{~V}, 0.03 \mathrm{nA}$ ) of c-P12 deposited via electrospray deposition and sublimed $\mathrm{C}_{60}$ (a: $0.3 \mathrm{ML}$; b: $0.7 \mathrm{ML}$ ). Scale bar $5 \mathrm{~nm}$.

We have also investigated $\mathrm{C}_{60}$ capture by the smaller $\boldsymbol{c}$-P12 nanorings. As for $\boldsymbol{c}$-P24 we first deposit the nanorings by electrospray (stacking is not observed for $\boldsymbol{c}$-P12 which are always found to have a monolayer height), followed by deposition of $\mathrm{C}_{60}$ by sublimation. Fig. 3 shows STM images acquired after the deposition of 0.3 ML and 0.7 ML of $\mathrm{C}_{60}$. For the lower coverage (Fig. 3a) we observe a preference for $\mathrm{C}_{60}$ adsorption in regions outside the nanoring ( $50 \%$ of nanorings are empty). In particular, we show that even where the local coverage of $\mathrm{C}_{60}$ is very high, within a hexagonally close packed island, $\mathrm{C}_{60}$ is not present within the nanoring. In fact for this particular nanoring there is no captured $\mathrm{C}_{60}$ and the nanoring is embedded within a fullerene island which has grown around it (note that the porphyrin sub-units of the nanoring can be resolved in this image). At higher coverage (Fig. $3 \mathrm{~b}$ ) we observe a lower fraction (28\%) of empty nanorings, and again see $\mathrm{C}_{60}$ islands grown around the nanoring. In this case, we resolve three $\mathrm{C}_{60}$ molecules within the nanoring.

The low number of captured $\mathrm{C}_{60}$ within the $\boldsymbol{c}$-P12 nanorings is consistent with the excluded region observed for the $\boldsymbol{c}$-P24. Since we expect a denuded region within $\sim 1.6 \mathrm{~nm}$ of the centre of the porphyrin macrocycle, the effective trapping radius of a nanoring is reduced from the geometric radius $(2.5 \mathrm{~nm})$ to $\sim 0.9 \mathrm{~nm}$. The effective area of a circular region with this radius is $\sim 2.5 \mathrm{~nm}^{2}$, which corresponds to $\sim 2.9 A_{\mathrm{mol}}$, where $A_{\mathrm{mol}}$ is the area occupied per $\mathrm{C}_{60}$ molecule in a hexagonally close-packed layer. This is consistent with our observation of a maximum of 3 captured $\mathrm{C}_{60}$ molecules.

The absence of $\mathrm{C}_{60}$ in $\boldsymbol{c}$-P12 at lower overall coverage implies that there are more favourable bonding sites available at low coverage. This is in contrast to $\boldsymbol{c}$-P24 and is, at least partially, due to the lower co-ordination of trapped molecules in $\boldsymbol{c}$-P12. Since only 3 molecules may be accommodated the maximum co-ordination is 2 , much lower than for the clusters in $\boldsymbol{c}$-P24 and elsewhere on the surface.

In conclusion we have found that the recently identified stacked cyclic porphyrin polymers can trap adsorbed material. The trapping probability shows a clear dependence on both the height of the nanorings and their perimeter length. Interestingly the geometry of the resulting nanostructures corresponds to cylindrical regions of one organic material enclosed within another organic adsorbate. In fact the molecules chosen here are two organic semiconductors and our observations motivate the formation of more ordered structures on alternative substrates which will allow a study of the influence of supramolecular order on optoelectronic properties such as exciton transfer, luminescence and photovoltaic effects. 
This work was supported by the UK Engineering and Physical Sciences (EPSRC) through grants EP/J006939/1 and EP/J007161/1.

\section{Notes and references}

1 J. A. Theobald, N. S. Oxtoby, M. A. Phillips, N. R. Champness and P. H. Beton, Nature, 2003, 424, 1029-1031.

2 S. Stepanow, M. Lingenfelder, A. Dmitriev, H. Spillmann, E. Delvigne, N. Lin, X. Deng, C. Cai, J. V Barth and K. Kern, Nat. Mater., 2004, 3, 229-233.

3 A. Stannard, J. C. Russell, M. O. Blunt, C. Salesiotis, M. D. C. Giménez-López, N. Taleb, M. Schröder, N. R. Champness, J. P. Garrahan and P. H. Beton, Nat. Chem., 2012, 4, 112-117.

4 L. Bartels, Nat. Chem., 2010, 2, 87-95.

5 J. V Barth, G. Costantini and K. Kern, Nature, 2005, 437, 671-679.

6 R. Madueno, M. T. Räisänen, C. Silien and M. Buck, Nature, 2008, 454, 618-621.

7 T. Kudernac, S. Lei, J. A. A. W. Elemans and S. De Feyter, Chem. Soc. Rev., 2009, 38, 402-421.

8 M. O. Blunt, J. C. Russell, M. D. C. Gimenez-Lopez, N. Taleb, X. Lin, M. Schröder, N. R. Champness and P. H. Beton, Nat. Chem., 2011, 3, 74-78.

9 J. Lu, S. Lei, Q. Zeng, S. Kang, C. Wang, L. Wan and C. Bai, J. Phys. Chem. B, 2004, 108, 5161-5165.
10 J. M. Macleod, O. Ivasenko, D. F. Perepichka and F. Rosei, Nanotechnology, 2007, 18, 424031.

11 S. J. H. Griessl, M. Lackinger, F. Jamitzky, T. Markert, M. Hietschold and W. M. Heckl, Langmuir, 2004, 20, 9403-9407.

12 M. O. Blunt, J. C. Russell, N. R. Champness and P. H. Beton, Chem. Commun., 2010, 46, 7157-7159.

13 M. C. O'Sullivan, J. K. Sprafke, D. V Kondratuk, C. Rinfray, T. D. W. Claridge, A. Saywell, M. O. Blunt, J. N. O'Shea, P. H. Beton, M. Malfois and H. L. Anderson, Nature, 2011, 469, 72-75.

14 D. V Kondratuk, L. M. A. Perdigao, M. C. O'Sullivan, S. Svatek, G. Smith, J. N. O'Shea, P. H. Beton and H. L. Anderson, Angew. Chem., Int. Ed., 2012, 51, 6696-6699.

15 S. A. Svatek, L. M. A. Perdigao, A. Stannard, M. B. Wieland, D. V. Kondratuk, H. L. Anderson, J. N. O'Shea and P. H. Beton, Nano Lett., 2013, 1-5.

16 S. Rauschenbach, F. L. Stadler, E. Lunedei, N. Malinowski, S. Koltsov, G. Costantini and K. Kern, Small, 2006, 2, 540-547.

17 A. Saywell, J. K. Sprafke, L. J. Esdaile, A. J. Britton, A. Rienzo, H. L. Anderson, J. N. O'Shea and P. H. Beton, Angew. Chem., Int. Ed., 2010, 49, 9136-9139.

18 M. Hutin, J. K. Sprafke, B. Odell, H. L. Anderson and T. D. W. Claridge, J. Am. Chem. Soc., 2013, 135, 12798-12807.

19 S. Guo, D. P. Fogarty, P. M. Nagel and S. A. Kandel, J. Phys. Chem. B, 2004, 14074-14081. 